\title{
STRONG RATIO LIMIT PROPERTY
}

\author{
BY STEVEN OREY 1
}

Communicated by M. Loève, August 8, 1961

1. Introduction. For every nonnegative integer $n$ let $p_{i j}^{(n)}$ be the $n$-step transition probabilities of a recurrent, irreducible, aperiodic Markov chain, $i, j=0,1, \cdots$. We say the chain has the strong ratio limit property $(S R L P)$ if there exist positive constants $\pi_{j}, j=0,1, \cdots$, such that

$$
\lim _{n \rightarrow \infty} \frac{p_{i j}^{(n+m)}}{p_{k h}^{(n)}}=\frac{\pi_{j}}{\pi_{h}}, \quad m=0, \pm 1, \pm 2, \cdots .
$$

It is well known that SRLP does not hold for all chains of the type considered here. ${ }^{2}$ We here present conditions for SRLP; the continuous parameter case is also considered.

2. Discrete parameter. Let ${ }_{k} p_{i j}^{(n)}=\operatorname{Prob}$ [going from $i$ to $j$ in $n$ steps without visiting $k$ at step number $1,2, \cdots, n-1]$. Note

$$
\sum_{n=1}^{\infty}{ }_{j} p_{i j}^{(n)}=1 \text { and g.c.d. }\left\{n: p_{i i}^{(n)}>0\right\}=1 \text { for every } i, j .
$$

LEMMA 1. SRLP holds if and only if $p_{00}^{(n+1)} / p_{00}^{(n)} \rightarrow 1$ as $n \rightarrow \infty$.

SKETCH OF PROOF. Assume $p_{00}^{(n+1)} / p_{00}^{(n)} \rightarrow 1$ as $n \rightarrow \infty$. For $n>N \geqq 1$ we have

$$
\begin{aligned}
A_{n}(\alpha) & =p_{0 \alpha}^{(n)} / p_{00}^{(n)}=\sum_{v=1}^{n} 0 p_{0 \alpha}^{(v)} p_{00}^{(n-v)} / p_{00}^{(n)}=\sum_{v=1}^{N}+\sum_{v=N+1}^{n} \\
& =B_{N, n}(\alpha)+C_{N, n}(\alpha) .
\end{aligned}
$$

Observe $A_{n}(\alpha)$ converges as $n \rightarrow \infty$ if and only if

$$
\lim _{N \rightarrow \infty} \lim _{n \rightarrow \infty} C_{N, n}(\alpha)
$$

1 This research was supported by the United States Air Force through the Air Force Office of Scientific Research and Development Command, under Contract No. AF-49(638)-617. Reproduction in whole or in part is permitted for any purpose of the United States Government.

2 For counterexample, general discussion and references to the literature see [1] under "ratio limit theorem, individual."

Frequently authors consider only the case $m=0$ in relation to (1). We do not know whether this is really more restrictive or not. 
exists. Setting $\alpha=0$ in (2.2) we see that the expression in (2.3) equals zerowhen $\alpha=0$. Let $j$ be an integer, depending on $\alpha$, such that ${ }_{0} p_{\alpha 0}^{(j)}>0$. For $\alpha \neq 0$ the estimate $0 p_{00}^{(n+\jmath)} \geqq_{0} p_{0 \alpha}^{(n)} 0 p_{\alpha 0}^{(j)}$ shows that (2.3) equals zero for every $\alpha$, proving the convergence of $A_{n}(\alpha)$. Similar arguments establish the lemma in full generality.

Let $u_{0}=1, u_{n}=p_{00}^{(n)}, f_{n}={ }_{0} p_{00}^{(n)}, n=1,2, \cdots$. We have the familiar renewal relation

$$
u_{n}=\sum_{k=1}^{n} f_{k} u_{n-k}, \quad n=1,2, \cdots .
$$

According to Lemma 1 SRLP is equivalent to

$$
u_{n+1} / u_{n} \rightarrow 1 \text { as } n \rightarrow \infty \text {. }
$$

We shall need the following simple lemma proved in [3]:

Lemma 2. If $\lim \sup \left(u_{n+1} / u_{n}\right) \leqq 1$ as $n \rightarrow \infty$ then (2.5) (and therefore $S R L P)$ holds.

Proof. Let $m=\lim \inf \left(u_{n+1} / u_{n}\right), M=\lim \sup u_{n+1} / u_{n}$ as $n \rightarrow \infty$. From (2.4) one gets easily

$$
m>\sum_{k=1}^{\infty} f_{k} M^{-k+1} .
$$

Thus if $M \leqq 1, m \geqq M$ so that $M=m=1$.

Theorem 1. If for some positive integer $m$

$$
\lim \sup u_{m(n+1)} / u_{m n} \leqq 1 \text { as } n \rightarrow \infty
$$

then (2.5) holds.

SkETCH OF PROOF. Assume (2.7). It is probabilistically evident that the sequence $\left\{u_{m n}\right\}, n=0,1, \cdots$ is also a "renewal sequence" for some persistent, aperiodic, recurrent event, so that Lemma 2 applies to give $u_{m(n+1)} / u_{m n} \rightarrow 1$ as $n \rightarrow \infty$. Further probabilistic arguments lead to $u_{m n+j} / u_{m n} \rightarrow 1$ as $n \rightarrow \infty$ for $j=1,2, \cdots, m-1 .^{3}$

The next theorem has several interesting probabilistic interpretations. However, the only proof we know is that given in our joint paper [3] which uses a fairly intricate analytic argument.

Theorem 2. Condition (2.5) is equivalent to

$$
\lim _{N \rightarrow \infty}\left[\sup _{n \geqq N} \frac{1}{u_{n}} \sum_{k=N}^{n} f_{k} u_{n-k}\right]=0 .
$$

3 A purely analytic proof of this theorem seems much harder. In [3] we give an analytic proof of a weaker theorem. 
3. Continuous parameter. Let $p_{i j}(t)$ be the transition probability matrix of a continuous parameter Markov chain, $t \geqq 0, i, j=0,1, \cdots$. We assume $p_{i j}(t) \rightarrow \delta_{i j}$ as $t \rightarrow 0$. We take the chain to be irreducible and recurrent. The SRLP now becomes

$$
\lim _{t \rightarrow \infty} \frac{p_{i j}(t+\Delta)}{p_{k h}(t)}=\frac{\pi_{j}}{\pi_{h}}, \quad-\infty<\Delta<\infty .
$$

We have

LEMma 3. If for some $\Delta>0$

$$
\lim _{t \rightarrow \infty} \frac{p_{00}(t+\delta)}{p_{00}(t)}=1 \text { uniformly for } 0 \leqq \delta \leqq \Delta
$$

then SRLP holds.

4. Reversible processes. The matrices $p_{i j}^{(n)}\left(p_{i j}(t)\right)$ of $\$ 1(\$ 2)$ belong to a reversible process if and only if $\pi_{i} p_{i j}=\pi_{j} p_{j i}\left(\pi_{i} p_{i j}(t)=\pi_{j} p_{j i}(t)\right.$ for all $t$ ) for every $i$ and $j$.

THEOREM 3. Reversibility implies SRLP, both in the discrete and continuous parameter case.

Proof. Assume reversibility. According to Kendall $[6 ; 7]$ we have the representation

$$
p_{i j}^{(n)}=\int_{-1}^{1} x^{n} d \Psi_{i j}(x), \quad n=0,1, \cdots,
$$

in the discrete parameter case and

$$
p_{i j}(t)=\int_{0}^{\infty} e^{-t x} d \Psi_{i j}(x), \quad t \geqq 0,
$$

in the continuous parmeter case, where the $\Psi_{i j}$ are real-valued functions of bounded variation, nondecreasing when $i=j$.

Formula (4.1) shows $u_{2 n}\left(=p_{00}^{(2 n)}\right)$ is nonincreasing so that SRLP follows from Theorem 1.

In the continuous parameter case the above argument gives us

$$
p_{00}((n+1) \delta) / p_{00}(n \delta) \rightarrow 1 \text { as } n \rightarrow \infty
$$

for every $\delta>0$. Formula (4.2) shows that $p_{00}(t)$ is nonincreasing. Thus condition (3.2) must hold, and Lemma 3 applies.

Representations like (4.1) and (4.2) have been used to establish SRLP in $[4 ; 5 ; 8]$. In previously treated cases however, the measures $\Psi_{i j}$ were known more explicitly and the arguments depend on detailed 
investigation of the behavior of these measures. In $[4 ; 5]$ there is also some discussion of SRLP in the transient case, which we have excluded. We do not know whether convergence of $p_{00}^{(n+1)} / p_{00}^{(n)}$ implies SRLP in the transient case.

\section{BIBLIOGRAPHY}

1. K. L. Chung, Markov chains with stationary transition probabilities, Berlin, Springer, 1960.

2. W. Feller, An introduction to probability theory and its applications, New York, Wiley, 1950.

3. A. Garsia, S. Orey, and E. Rodemich, Asymptotic behavior of successive coeffcients of some power series, to appear.

4. S. Karlin and J. McGregor, The classification of birth and death processes, Trans. Amer. Math. Soc. vol. 86 (1957) pp. 366-400.

5. — Random walks, Illinois J. Math. vol. 3 (1959) pp. 66-81.

6. D. G. Kendall, Unitary dilations of Markov transition operators, and the corresponding integral representation for transition-probability matrices, Harold Cramer Volume (Ed. U. Grenander) Stockholm (1960) pp. 139-161.

7. - Unitary dilations of one-parameter semigroups of Markov transition operators, and the corresponding integral representations for Markov processes with a countable infinity of states, Proc. London Math. Soc. vol. 9 (1959) pp. 417-431.

8. W. Pruitt, Bilateral birth and death processes, ONR Technical Report No. 22, Contract Nonr-225(28) (NR-047-019), Applied Math. and Stat. Lab., Stanford, (1960).

UNIVERSITY OF MINNESOTA 